Research Paper

\title{
Reduction of pl-CSA through ChSy-2 knockout inhibits tumorigenesis and metastasis of choriocarcinoma in JEG3 cells
}

Juzuo Zhang1,2\#, Zhilong Chen²,3\#, Baobei Wang², Jie Chen², Tianxia Xiao ${ }^{2}$, Jian V. Zhang ${ }^{2}$, Shiling Chen ${ }^{4^{凶}}$ and Xiujun Fan ${ }^{\circledR}$

1. College of Biological and Food Engineering, Huaihua University, Huaihua, Hunan 418000, China

2. Center for Reproduction and Health Development, Shenzhen Institutes of Advanced Technology, Chinese Academy of Science, Shenzhen, Guangdong 518055, China.

3. College of Veterinary Medicine, Hunan Agricultural University, Changsha, Hunan.

4. Department of Gynecology and Obstetrics, Southern Medical University, Guangzhou, Guangdong 510515, China.

\#These authors contributed equally to this study.

$\triangle$ Corresponding authors: Dr. Xiujun Fan or Dr. Jian v Zhang, Center for Reproduction and Health Development, Shenzhen Institutes of Advanced Technology, Chinese Academy of Science, 1068 Xueyuan Avenue, Shenzhen University Town, Shenzhen, Guangdong 518055, P.R. China. E-mail: xiujun.fan@gmail.com or E-mail: jian.zhang@siat.ac.cn. Prof. Shiling Chen, Department of Gynecology and Obstetrics, Southern Medical University, 1023-1063, Shatai South Road, Baiyun District, Guangzhou, Guangdong 510515,P.R. China. E-mail: chensl_92@163.com.

(c) The author(s). This is an open access article distributed under the terms of the Creative Commons Attribution License (https://creativecommons.org/licenses/by/4.0/). See http:/ /ivyspring.com/terms for full terms and conditions.

Received: 2020.08.12; Accepted: 2020.10.07; Published: 2021.01.01

\begin{abstract}
Background: Placental-like chondroitin sulfate A (pl-CSA) is exclusively expressed in cancerous and placental tissues and is highly correlated with the degree of malignancy. However, the mechanism through which pl-CSA regulates tumorigenesis and metastasis in choriocarcinoma remains unclear.

Methods: Stable transfectants of the JEG3 choriocarcinoma cell line, including a negative control (NC) line and a cell line with knockout of the biosynthetic enzyme CS synthase-2 (ChSy-2) (ChSy-2-/-), were obtained using CRISPR/Cas9 systems and identified by immunofluorescence, flow cytometry, western blots and enzyme-linked immunosorbent assays (ELISAs). The proliferation, migration, invasion and colony formation of the cells were determined by a cell counting kit, scratch-wound assays, transwell assays and soft agar colony formation assays in vitro, respectively. The tumorigenesis and metastasis of choriocarcinoma were also investigated through two xenograft models in vivo.

Results: The ChSy-2 protein in the ChSy-2--group was below the detection threshold, which was accompanied a significant reduction in the pl-CSA level. Reducing pl-CSA through ChSy-2 knockout significantly inhibited cell proliferation, migration, invasion and colony formation in vitro and tumorigenesis and metastasis of choriocarcinoma, with deceases in tumor volume and metastatic foci and a high percent survival compared to the NC in vivo.

Conclusion: pl-CSA, as a necessary component of JEG-3 cells, was efficiently reduced through ChSy-2 knockout, which significantly inhibited the tumorigenesis and metastasis of choriocarcinoma. ChSy-2/pl-CSA could be alternative targets for tumor therapy.
\end{abstract}

Key words: choriocarcinoma, ChSy-2/pl-CSA, tumorigenesis, molecular therapy

\section{Introduction}

Cancer is a serious public health problem worldwide, and choriocarcinoma originating from the trophoblastic tissue in gestation or without pregnancy severely endangers females and even males. The incidence of choriocarcinoma varies in different regions, ranging from 1 in 15,000-50,000 pregnancies in Europe and North America to 1 in 500-20,000 pregnancies in Southeast Asia [1], and is $>5$ in 10,000 pregnancies in China [2]. Although choriocarcinoma is relatively rare, case reports have described it in 
females and males or from carcinoma in situ to metastasis [3]. Patients with choriocarcinoma range from 17 to 67 years old, and the asymptomatic period varies from 4 weeks to 25 years [4]. Although the prognosis of choriocarcinoma is relatively good, its effects, including damage to multiple organs or injuries and pain accompanying chemotherapy, are harmful and approximately $40 \%$ of pregnancies with choriocarcinoma result in neonatal mortality [5]. Choriocarcinoma, a highly malignant epithelial cell tumor [6], is mainly characterized by immortalized trophoblast cells with uncontrolled proliferation, migration and invasion and distant metastasis to the blood vessels, lung, liver or brain [7-10]. Currently, the molecules involved in the tumorigenesis and metastasis of trophoblast cells are unclear, which is a major challenge to diagnosis and treatment. Due to the lack of reliable target biomarkers, the main therapeutic approach to choriocarcinoma is chemotherapy based on pathological examination and human chorionic gonadotropin (hCG) levels [11] rather than surgery or immunotherapy. Therefore, the degree of malignancy and various clinical phenotypes of choriocarcinoma need to be investigated, and identification of target genes related to occurrence and development, clinical diagnosis and molecular therapy is urgently needed.

Placental-like chondroitin sulfate A (pl-CSA) is a distinct and low-sulfated subtype of chondroitin sulfate (CS), a glycosaminoglycan (GAG) that plays a vital role in tumorigenesis. This receptor can specifically bind with VAR2CSA of Plasmodium falciparum-infected erythrocytes or various other ligands and has been associated with the degree of malignancy $[12,13]$. With its typical GlcA-GalNAc (4S) disaccharide unit and relatively low variation, pl-CSA is synthesized in multiple types of cancer $[13,14]$ by chondroitin sulfate synthase (ChSy, especially ChSy-2) and is covalently linked to its core protein [15]. As the biosynthetic enzyme of pl-CSA, ChSy-2 can induce high expression of CSA in placental and tumor tissues and may be a key glycosyltransferase [16-18]. Targeting ChSy-2 may be an important method to study pl-CSA biofunction because there is no efficient way to directly study glycobiology. Thus, ChSy-2/pl-CSA may be promising cancer biomarkers and play an important role in tumorigenesis and metastasis.

To investigate the role of ChSy-2/pl-CSA in choriocarcinoma, we decreased the pl-CSA content in JEG3 choriocarcinoma cells by deleting the biosynthetic enzyme ChSy-2. The modified JEG3 cells were identified through immunofluorescence, flow cytometry, western blot and enzyme-linked immunosorbent assays (ELISAs). The properties of modified JEG3 cells, including proliferation, migration, invasion and colony formation in soft agar, were investigated through Cell Counting Kit 8 (CCK-8), wound-healing, transwell, and soft agar colony assays, respectively. The roles of ChSy/pl-CSA in the tumorigenesis and metastasis of choriocarcinoma were also investigated using two xenograft mouse models in vivo.

\section{Materials and methods}

\section{Cell culture}

The human choriocarcinoma cell line JEG3 was obtained from the American Type Tissue Collection (ATCC number: HTB-36TM, Manassas, VA) and was authenticated using short tandem repeat (STR) analysis for mycoplasma contamination. The cells were routinely cultured in DMEM/F12 (cat. no. 11320033; Thermo Fisher Scientific, Inc., Waltham, MA, USA) supplemented with $10 \%$ fetal bovine serum (cat. no. 16000044; Thermo Fisher Scientific, Inc.), penicillin $(100 \mathrm{U} / \mathrm{ml})$ and streptomycin (100 $\mu \mathrm{g} / \mathrm{ml}$ ) (cat. no. 15140122; Thermo Fisher Scientific, Inc.) and maintained in a humidified $5 \% \quad \mathrm{CO}_{2}$ atmosphere at $37^{\circ} \mathrm{C}$.

\section{Stable transfection}

Stable transfectants were obtained from the JEG3 cell line, including a negative control line (NC) using an empty lentivirus CRISPR/Cas v2 vector with the puromycin resistance gene and a ChSy-2 knockout line (ChSy-2/-) using a plasmid inserting a gRNA of GCCGCGCGGCAACACCAACG (according to online resources at the Zhang Lab website: https://zlab. bio/guide-design-resources) into a vector for targeting exon 1 of $\mathrm{ChSy}-2$ in the coding sequence (AB175496.1).

Plasmid transfection was performed with Lipofectamine ${ }^{\circledR} 3000$ Reagent (cat. no. L3000008; Thermo Fisher Scientific, Inc.) according to the manufacturer's instructions when the cultured cells reached approximately $70 \%$ confluence. The transfected JEG3 cells were screened in medium containing $4 \mu \mathrm{g} / \mathrm{ml}$ puromycin (cat. no. anti-Pr-1, InvivoGen) for 1 week. The cells were isolated, amplified and maintained in $2 \mu \mathrm{g} / \mathrm{ml}$ puromycin for subsequent experiments.

\section{Preparation of rVAR2}

For analysis of pl-CSA, a recombinant VAR2CSA labeled with a $6 \times$ His tag (rVAR2), specifically interaction with pl-CSA, was expressed by inserting this fragment into a pET28a $(+)$ vector that was transformed into Escherichia coli BL21, and was purified using a $\mathrm{Ni}^{2+}$ affinity column (cat. no. DP101-01; Transgen Biotech Co., Ltd., Beijing, China) 
from the supernatant of bacterial culture. The purified rVAR2 was used in the following experiments.

\section{Immunofluorescence and flow cytometry}

The cultured cells were subjected to immunofluorescence and flow cytometry. Briefly, after fixation, permeabilization and blockage, the cells were successively incubated with a mixture of ChSy-2 rabbit primary antibody (dilution, 1:500; cat. no. ab75052, Abcam) and rVAR2-6 $\times$ His recombinant protein (dilution, 1:200; Figure 1), and a mixture of FITC-labeled goat anti-rabbit (dilution, 1:1000; cat. no. ab6717; Abcam) and DyLight ${ }^{\circledR}$ 650-labeled anti-6 6 His tag secondary antibodies (dilution, 1:1000; cat. no. ab117504, Abcam), and followed by counterstain with $0.4 \mu \mathrm{g} / \mathrm{ml}$ DAPI (cat. no. AR1177, Wuhan Boster Biological Technology, Ltd., Wuhan, China). Stained cells were observed and analyzed under a fluorescence microscope (BX53; Olympus Corporation, Tokyo, Japan) using Image-Pro Plus software version 6.0 (Media Cybernetics, Inc., Rockville, MD, USA), and were also analyzed by flow cytometry (CytoFLEX S; Beckman Coulter, Inc., Brea, CA, USA) using FlowJo software (FlowJo 7.6.1; Treestar, Inc., Ashland, OR, USA).

\section{Proliferative analysis}

Cell proliferation was detected using CCK-8 assays [19]. Briefly, the cells were seeded into 96-well plates (100 $\mu \mathrm{l} /$ well; 2,500 cells/well) and were cultured at $37^{\circ} \mathrm{C}$ in an environment with $5 \% \mathrm{CO}_{2}$. At $12,24,36,48,60,72$ and $84 \mathrm{~h}, 10 \mu \mathrm{l}$ of CCK-8 solution (cat. no. CK04; Dojindo Molecular Technologies, Inc., Kumamoto, Japan) was added to each well, followed by incubation for $3 \mathrm{~h}$, and the solution color was monitored. When the difference in the color was significant between groups, absorbance was measured at $450 \mathrm{~nm}$.

\section{Cell migration assay}

Cell migration was detected using a scratchwound assay [20]. Briefly, the $90 \%$ confluent cell layer was scratched using a $10-\mu l$ pipette tip, and detached cells were removed by washing with PBS. The cells were cultured in medium with 2\% FBS for $48 \mathrm{~h}$. Images of the scratch wounds were obtained using an inverted phase-contrast microscope (BX53, Olympus Corporation, Tokyo, Japan) equipped with a digital camera at 0,24 and $48 \mathrm{~h}$. The wound width was determined using Image-Pro Plus software version 6.0 .

\section{Cell invasion assay}

Cell invasion was detected using a transwell assay [21] with a Corning ${ }^{\circledR}$ BioCoat $^{\mathrm{TM}}$ Matrigel ${ }^{\circledR}$ Invasion Chamber (cat. no., 354480; Corning, Inc., NY,
USA). Briefly, the cells were seeded into the transwell upper chamber at a density of $5 \times 10^{4}$ cells/well in 200 $\mu \mathrm{l}$ of serum-free medium; $600 \mu \mathrm{l}$ of medium with $10 \%$ FBS was added to the lower chambers. Following incubation for $24 \mathrm{~h}$, the noninvasive cells on the upper membrane of the insert were removed using a cotton swab. The migrated cells adhered to the lower membrane surface were fixed and stained with crystal violet dye, and the number of cells was then counted under a microscope in 5 random optical fields.

\section{Soft agar colony formation assay}

The soft agar colony formation assay was used to determine carcinogenesis and malignant transformation in vitro [22]. Briefly, the cells $\left(10^{5}\right.$ cells/well) were mixed with $0.3 \%$ agar dissolved in growth medium and plated on top of a bottom layer of $0.7 \%$ agar dissolved in growth medium in a 6-well plate. A layer of complete medium $(\sim 200 \mu \mathrm{l})$ was maintained above the upper agar layer and changed every 3 days. After 26 days, the colonies were dyed with crystal violet dye ( $0.01 \%$ solution). Images of the clones were observed using an inverted phasecontrast microscope (BX53, Olympus Corporation, Tokyo, Japan) equipped with a digital camera. The number of clones was determined using Image-Pro Plus software version 6.0.

\section{Tumorigenesis and metastasis of modified cells in vivo}

Cell tumorigenesis and metastasis were detected using in vivo imaging systems (IVIS, Xenogen Corporation, CA, USA) [23]. The cells were engineered to constitutively express the firefly luciferase reporter gene (Fluc2). Then, $1 \times 10^{6}$ Fluc2carrying JEG3 cells (JEG3-Fluc2) in $100 \mu$ of PBS were injected into specific-pathogen-free (SPF) 4-6-weekold female nude mice (BALB/c background) either subcutaneously for tumorigenesis or through the tail vein for metastasis. For determination of tumor growth conditions, mice carrying JEG3-Fluc2 cells were intraperitoneally administered $150 \mathrm{mg} / \mathrm{kg}$ body weight D-luciferin (cat. no., 103404-75-7; Gold Biotechnology, Inc., St Louis, MO, USA), and the cells emitted a visual light signal that was monitored using IVIS Spectrum 2 days after cell administration and then again 4 days later. The photon flux from the mouse was proportional to the number of lightemitting cells, and the signal was measured to monitor tumorigenesis and metastasis. The region of interest (ROI) of fluorescence imaging was analyzed with an IVIS imaging system. Tumor volume was measured as follows: tumor volume $=$ (tumor length) $x$ (tumor width $)^{2} / 2$. The humane endpoint was based on the maximum acceptable total burden of animal 
models, no longer than 60 days. Animals of all groups in the tumorigenesis experiment were euthanized when the measured volume of subcutaneous tumors was over $1 \mathrm{~cm}^{3}$ in any group. Animals in all groups in the metastasis experiment were euthanized on the same day when metastasis was observed in one or two organs in any group. Moreover, the health of the mice was monitored three times a week, with no obvious pain or distress [24].

\section{Serum hCG measurement}

Blood samples were collected from mice administered JEG3 cells through the orbital venous system after anesthesia with isoflurane. The serum was separated from blood samples by centrifugation. Serum hCG levels were determined by radioimmunoassays using an $\mathrm{XH} 6080$ radioimmunity analyzer, carried out by Beijing North Institute of Biotechnology Co., Ltd. (Beijing, China).

\section{ELISA detection of pl-CSA}

The pl-CSA level in JEG3 cells was detected as described previously [14]. Briefly, approximately $10^{6}$ well-cultured cells/ml were collected and lysed in lysis buffer for pl-CSA detection. The lysate was added to 96-microwell plates precoated with pl-CSA-BP capture protein, successively followed by incubation with anti-pl-CSA antibody, horseradish peroxidase (HRP)-labeled secondary antibody, and substrate solution $\left[3,3^{\prime}, 5,5^{\prime}\right.$-tetramethylbenzidine (TMB)]. After the reaction was stopped with $\mathrm{H}_{2} \mathrm{SO}_{4}$, the absorbance was recorded at $450 \mathrm{~nm}$ (OD450 nm) to calculate the pl-CSA content.

\section{Protein extraction and western blot analysis}

Protein extracts were prepared from cells using M-PER ${ }^{\mathrm{TM}}$ Mammalian Protein Extraction Reagent (cat. no. 78503; Thermo Fisher Scientific, Inc.) supplemented with EDTA-free complete protease inhibitor cocktail (cat. No. 88265; Thermo Fisher Scientific, Inc.). Protein content was determined using a Micro BCA ${ }^{\mathrm{TM}}$ Protein Assay Kit (cat. no. 23235; Thermo Fisher Scientific, Inc.). Protein samples $(30 \mu \mathrm{g})$ were separated on a 12\% SDS-PAGE gel and electrophoretically transferred to PVDF membranes (cat. no. 162-017; Bio-Rad Laboratories, Inc., Hercules, CA, USA). After blockage, the membranes were successively incubated with anti-human ChSy-2 or $\beta$-actin rabbit antibodies (dilution, 1:1000, ab75052 or ab16039, respectively; Abcam) and goat anti-rabbit IgG antibody conjugated to horseradish peroxidase (dilution, 1:5000; cat. no. ab97051; Abcam). Protein bands were visualized by enhanced chemiluminescence using SuperSignal ${ }^{\mathrm{TM}}$ West Pico Chemiluminescent Substrate (Thermo Fisher Scientific, Inc.) on a ChemiDoc ${ }^{\mathrm{TM}}$ XRS+ system (Bio-Rad
Laboratories, Inc.). Target blots were normalized to $\beta$-actin and semiquantified by densitometry using ImageJ software (v2.1.4.7; National Institutes of Health, Bethesda, MD, USA).

\section{Statistical analysis}

Data are presented as the mean \pm standard deviation of at least three independent experiments. The variance between all groups was assessed by ANOVA, and the statistical significance of the differences between groups was assessed using Tukey's multiple comparisons test. Statistical analysis was performed using SPSS software version 19.0 (IBM Corp., Armonk, NY, USA). ** $P<0.01$ and * $P<0.05$ were considered significant.

\section{Results}

\section{ChSy-2 knockout significantly reduces the pl-CSA content in JEG 3 choriocarcinoma cells}

Through prokaryotic expression and affinity purification, the electrophoretically pure rVAR2 was obtained for analysis of pl-CSA (Figure 1). The protein levels of ChSy-2 and contents of pl-CSA in the transfectants of positive cells and negative cells were determined by immunofluorescence, flow cytometry, western blots and ELISAs. Compared with that in the NC group, the green fluorescence density (ChSy-2) was below the threshold, and the red fluorescence density (pl-CSA) was significantly decreased in the ChSy-2\% group (Figure 2A and B). The ChSy-2 protein level was below the threshold (Figure 2C), and the pl-CSA content in the ChSy-2\% group was significantly decreased (Figure 2D) compared with that in the NC group. According to these data, ChSy-2, the biosynthetic enzyme of pl-CSA, was successfully eliminated in the ChSy-2/- group, and ChSy-2 knockout reduced the pl-CSA content in JEG3 cells.

\section{Reducing pl-CSA through ChSy-2 knockout impedes the functions of JEG3 cells in vitro}

The proliferation, migration, invasion and colony formation capacities of the JEG3 cells were investigated by CCK-8, scratch-wound, transwell and soft agar colony assays, respectively. Compared with that in the NC group, the proliferation curve of the ChSy-2\% cells maintained a flat trend (Figure 3D), and the migration rate of the ChSy-2\% cells was significantly decreased (Figure 3A and E), and the number of invading cells was significantly lower (Figure 3B and $\mathrm{F}$ ), and the number of clones was significantly lower (Figure $3 \mathrm{C}$ and G). According to these data, downregulation of ChSy-2/pl-CSA significantly impeded JEG3 cell proliferation, migration, invasion and colony formation in vitro. 


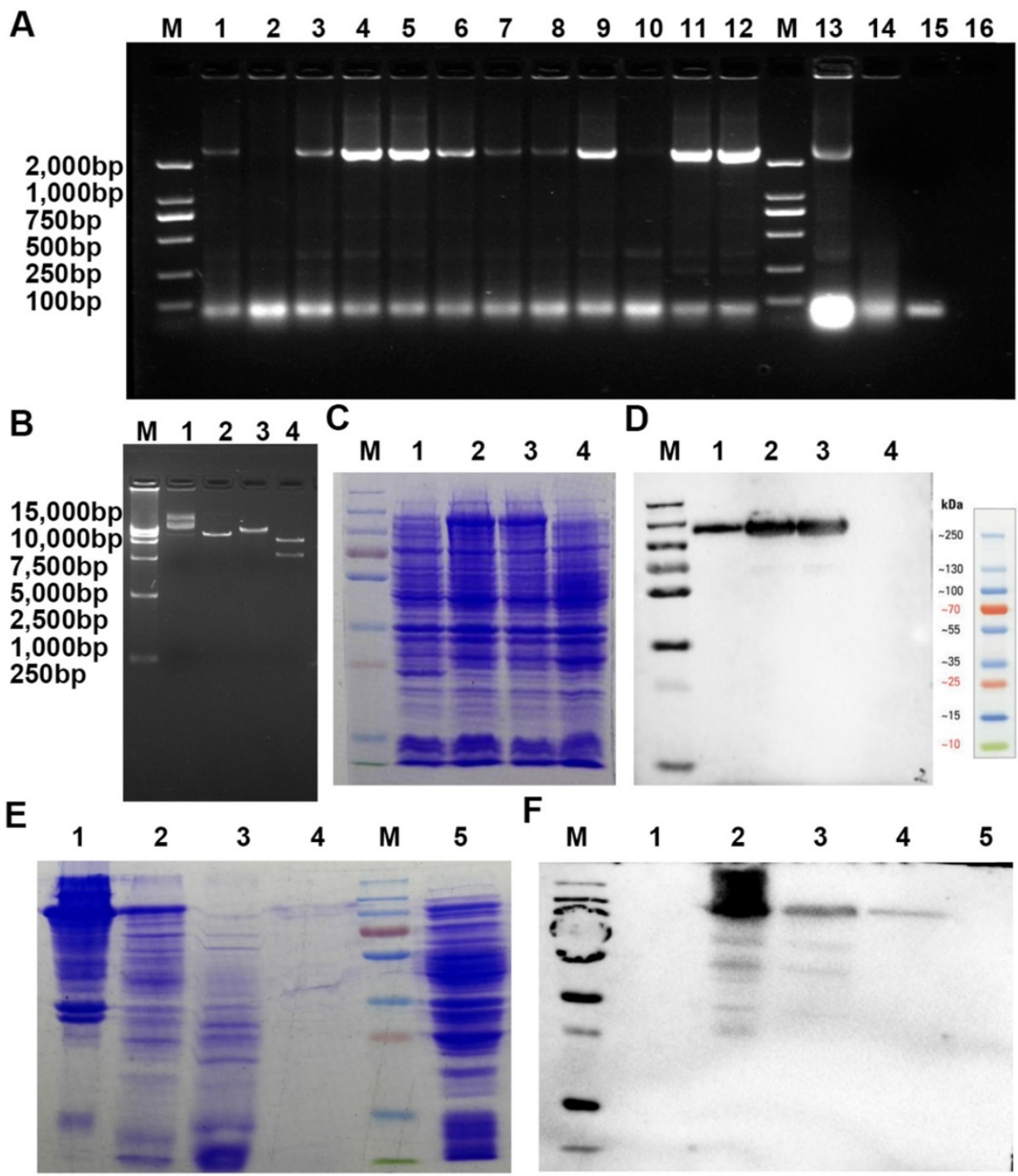

Figure 1. A recombinant VAR2CSA labeled with a $6 \times$ His tag (rVAR2), specifically binding pl-CSA, was expressed and purified from E. coli BL21 by inserting this fragment into a pET28a(+) plasmid that was then transferred into E. coli. BL21. A. Screening of positive clones by PCR amplification (1-16, different clones amplified with PCR primers; M, DNA marker). B. Determination of the plasmid using restriction enzyme digestion (1 and 2, uncut plasmid; 3, plasmid digestion with BamHI; 4, plasmid digestion with BamHI and Sall; M, DNA marker). C and D. Determination of rVAR2 expression in the bacterial extract by reducing SDS-PAGE, followed by (C) Coomassie blue staining and (D) western blot analysis with total protein extract from (1-3) different positive clones and (4) the negative control. E and F. Determination of purification of rVAR2 using reduced SDS-PAGE, followed by Coomassie blue staining (E: 1, positive clone bacterial pellet; 2 , bacterial culture supernatant following lysis and centrifugation; 3 , unbound components; 4 , bound components from the His tag column; 5 , negative bacterial pellet; $M$, protein marker) and western blot analysis (F: 1 , unbound components; 2 , positive clone bacterial pellet; 3 , bacterial culture supernatant following lysis and centrifugation; 4, bound components from His tag column; 5 , negative bacterial pellet; $M$, protein marker) with total protein extract.

\section{Reducing pl-CSA through ChSy-2 knockout inhibits tumorigenesis and metastasis of choriocarcinoma in vivo}

Following subcutaneous injection, the luciferase signals produced by the JEG3-Fluc2 cells were monitored in a tumorigenesis model. The tumor volume was continuously enlarged in the NC group over time. Interestingly, the tumor remained small in the ChSy-2/- group until the end of the experiment, and the percent survival in the ChSy-2\%- group was significantly higher than that in the NC group (Figure $4 A, B$ and G). Following tail vein injection, the luciferase signals produced by the JEG3-Fluc2 cells were monitored in a metastasis model. The luciferase signal was mainly located in the lungs for the first 10 days, with an insignificant difference, suggesting that the cancer cells were mainly in the lungs at the early stage in both the NC and ChSy-2/- groups. After 16 days, the luciferase signals in the NC group were significantly increased and transferred to the liver and kidney, suggesting that metastasis had occurred. Interestingly, the luciferase signals in the ChSy-2-/cells remained constant and even decreased (Figure 4C-E). Moreover, the serum hCG level in the ChSy-2-/mice was significantly lower than that in the NC mice (Figure 4F). Therefore, downregulation of ChSy-2/plCSA significantly inhibited tumorigenesis and metastasis of choriocarcinoma in vivo. 
A
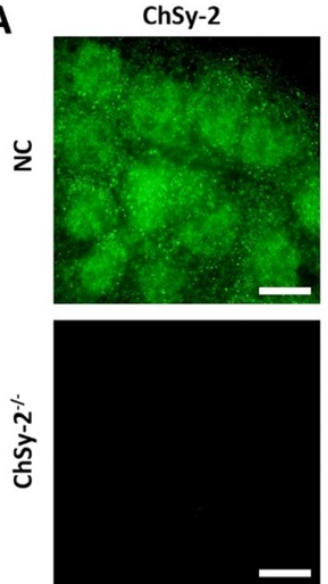

B

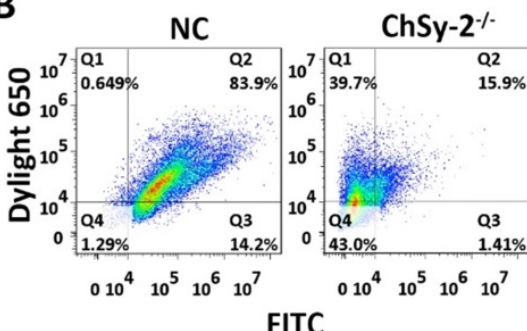

pl-CSA
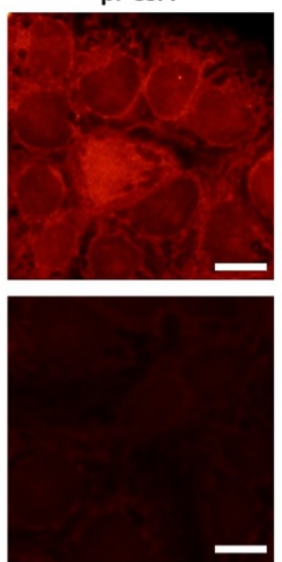

C

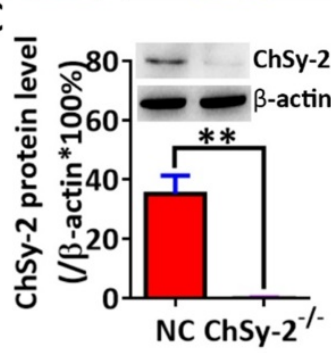

Merge
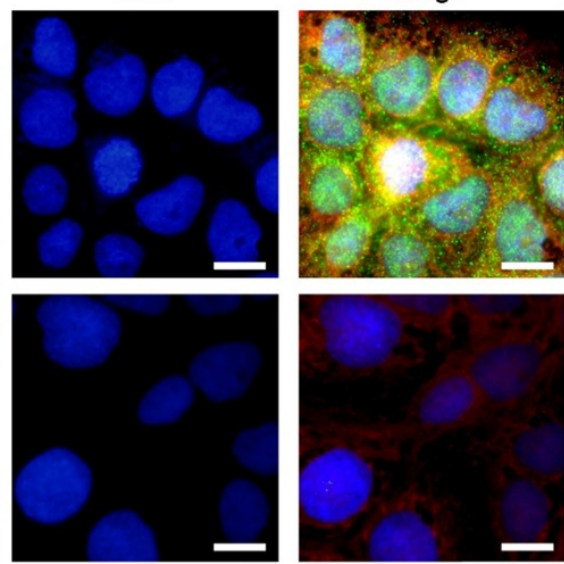

D

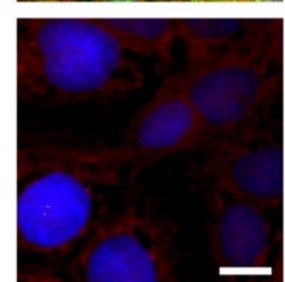

D.

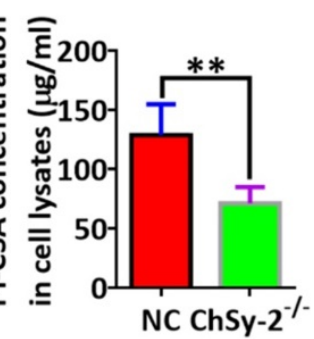

Figure 2. The pl-CSA content was significantly downregulated through ChSy-2 knockout. A. Immunofluorescence staining (green for ChSy-2, red for pl-CSA, blue for the nucleus, merged for color composition). Scale bar, $20 \mu \mathrm{m}$. B. Flow cytometry with FITC (the maximum absorption wavelength is $495 \mathrm{~nm}$, and the maximum emission wavelength is $519 \mathrm{~nm}$ ) and DyLight $\AA 650$ (the maximum absorption wavelength is $652 \mathrm{~nm}$, and the maximum emission wavelength is $672 \mathrm{~nm}$ ) representing ChSy-2 and pl-CSA, respectively. C. Western blot and analysis of integral optical density based on the protein band compared with $\beta$-actin. D. ELISA detection and analysis of pl-CSA content in choriocarcinoma. ${ }^{* * P}<0.01$. pl-CSA, placental-like chondroitin sulfate A; ChSy-2, biosynthetic enzyme CS synthase- 2 .

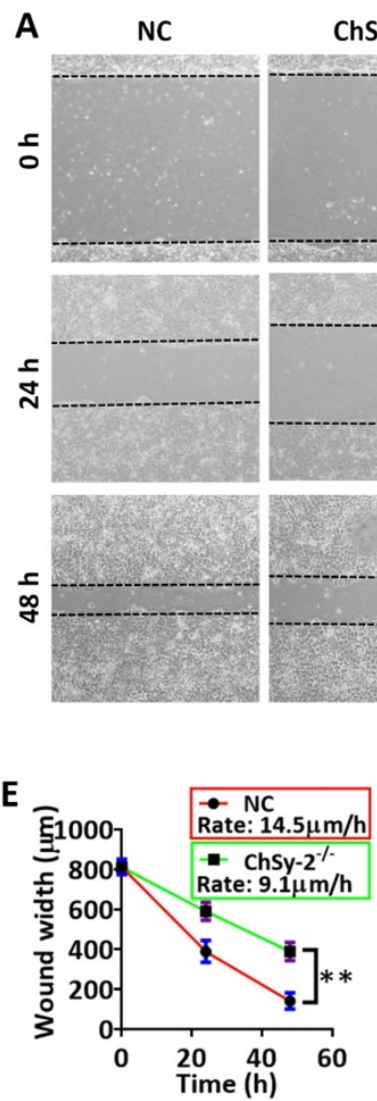

ChSy-2\%

\section{B}

守

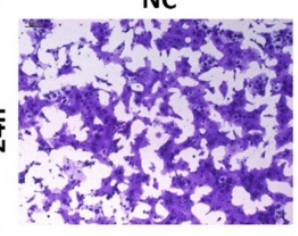

NC

NC

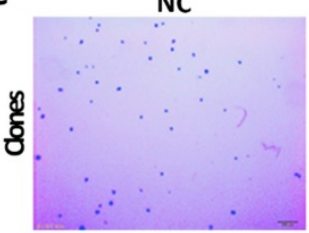

D

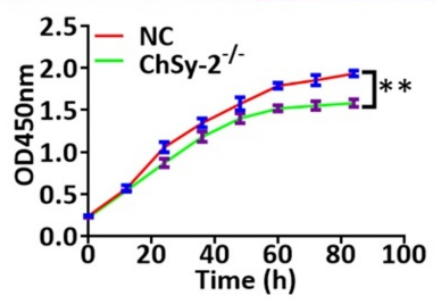

$\mathbf{F}$

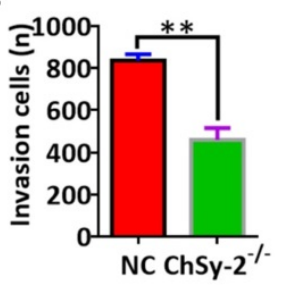

ChSy-2 $\%$

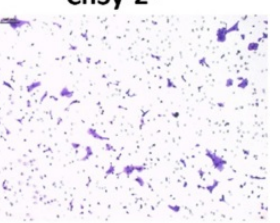

ChSy-2\%

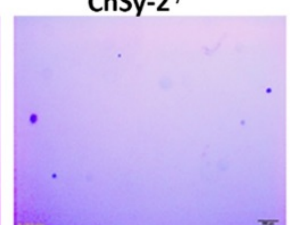

$\mathbf{G}$

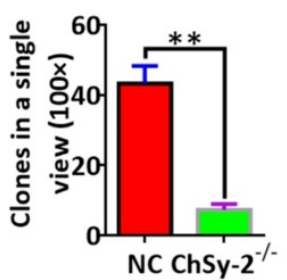

Figure 3. Reducing pl-CSA through ChSy-2 knockout significantly impeded cell proliferation, migration, invasion and colony formation in vitro. A. Scratch-wound assay for cell migration at 0, 24 and $48 \mathrm{~h}$. Scale bar, $100 \mu \mathrm{m}$. B. Transwell assay for cell invasion at $24 \mathrm{~h}$. Scale bar, $100 \mu \mathrm{m}$. C. Soft agar colony formation assay for colony formation in vitro. Scale bar, $100 \mu \mathrm{m}$. D. Cell proliferation index curve using Cell Counting Kit- 8 assays. E. The analysis of the scratch-wound width and migration ratio (distance of cell migration at $1 \mathrm{~h}$ ) of cells based on scratch-wound assays for $48 \mathrm{~h}$. F. The analysis of invading cell number. G. Analysis of clone numbers in soft agar assays for tumorigenesis. $* * P<0.01$. pl-CSA, placental-like chondroitin sulfate A; ChSy-2, biosynthetic enzyme CS synthase-2. 
A

2d

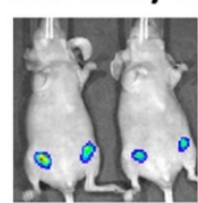

10d

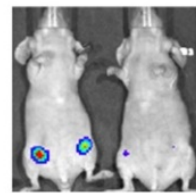

18d

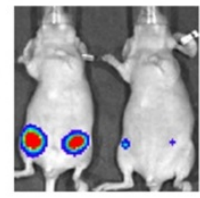

26d

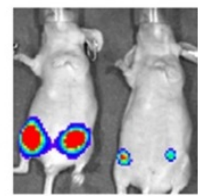

C
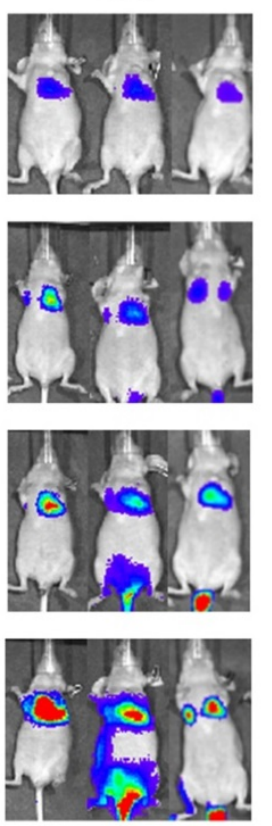

B

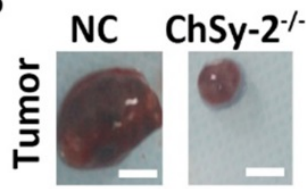

D

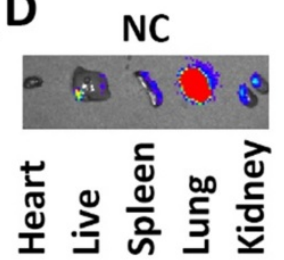

ChSy-2\%
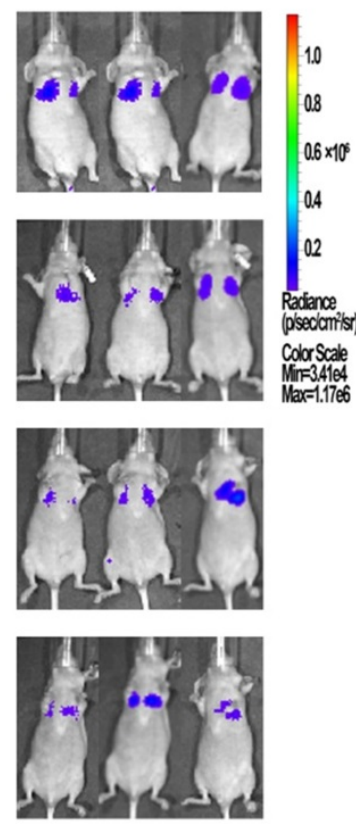

ChSy-2 ${ }^{-}$

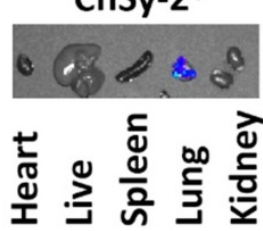

E

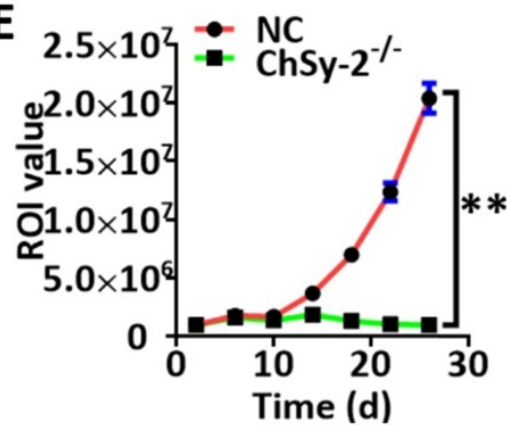

$\mathbf{F}$

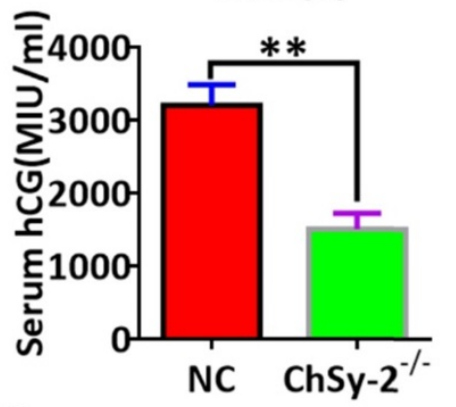

$G$

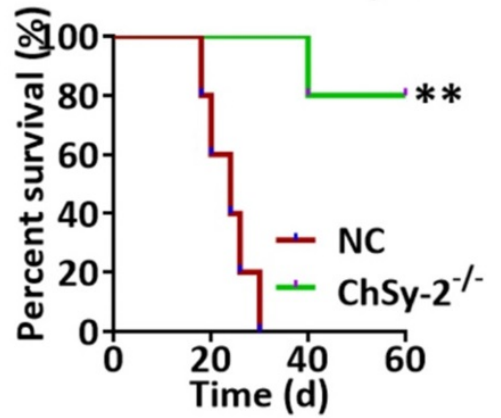

Figure 4. Reducing pl-CSA through ChSy-2 knockout significantly inhibited tumorigenesis and metastasis of choriocarcinoma in vivo. A. Monitoring of subcutaneous tumorigenesis through the IVIS imaging system with D-luciferin at 2, 10,18 and $26 \mathrm{~d}$ in vivo. B. Volume detection of subcutaneous tumors. Scale bar, $0.5 \mathrm{~cm}$. C. Monitoring of tumor metastasis after tail vein injection of modified JEG3 cell lines through the IVIS imaging system with D-luciferin at 2, 10,18 and $26 \mathrm{~d}$ in vivo. D. The mouse models were euthanized, and the visceral organs, including the heart, liver, spleen, lung, and kidney, were observed for the fluorescence of metastatic tissues ex vivo. E. The region of interest (ROI) analysis of the whole body for tumor metastasis. F. Serum hCG levels. G. Percent survival of mice with subcutaneous tumorigenesis. $* * P<0.01$. Pl-CSA, placental-like chondroitin sulfate A; ChSy-2, biosynthetic enzyme CS synthase-2. hCG, human chorionic gonadotropin.

\section{Discussion}

Choriocarcinoma has a high cure rate following chemotherapy with high-dose drug delivery, but it is associated with increased patient suffering [25]. It remains a very promising approach to explore biotherapy targets. A previous study demonstrated that pl-CSA-targeted nanoparticles loaded with low-dose doxorubicin strongly inhibited the tumorigenesis and metastasis of choriocarcinoma in vitro and in vivo [26], suggesting that targeting pl-CSA could be a promising treatment for choriocarcinoma. However, the intrinsic function of pl-CSA in the tumorigenesis and metastasis of choriocarcinoma remains unclear. In this study, we further demonstrated that the pl-CSA was a promising biomarker for choriocarcinoma treatment, and the content of pl-CSA in tumor tissues might imply grade malignancy. Pl-CSA content could be efficiently reduced by targeting its biosynthetic enzyme ChSy-2, suggesting a promising approach to treat choriocarcinoma through inhibiting ChSy-2/pl-CSA pathway.

Glycosylation, a post-translational modification, exists in various biological processes, including physiological and pathological processes, in which the attachment of sulfated glycans to proteins plays an important role in biological functions and carcinogenesis [27]. The conventional CSs, as sulfated GAGs, are assembled in Golgi compartments, and CS chain is covalently attached to its core proteins CSPGs, first through a tetrasaccharide linkage region linked to specific serine residues of the core protein and then by ligation with typical disaccharides [15]. The tetrasaccharide linkage region is a general structure for a series of CS subtypes, as well as for some subtypes of heparin, dermatan and hyaluronic acid [15]. The diverse CS chains are classified into CSA, CSB and CSC that depends on properties of disaccharide units by the corresponding specific 
glycosyltransferase and sulfotransferases, such as chondroitin synthase (ChSy, including ChSy-1, ChSy-2 and ChSy-3) and chondroitin polymerizing factor [15]. Pl-CSA with specific and distinctive disaccharide units is specifically expressed in placental [28] and cancerous tissues [13]. The structural properties of disaccharide units might facilitate pl-CSA interaction with placental or cancer bioactive molecules, thus for its function. However, it needs to further investigate the exact relationship between the pl-CSA and interacting molecules. Moreover, a presumed model of pl-CSA spatiotemporal expression might also happen in different stage of cancer development that might play a key role in tumorigenesis and metastasis of choriocarcinoma.

Targeting metabolic enzymes to study biopolysaccharides is an important technique, and metabolic enzymes were proposed as indirect targets for studying pl-CSA function. However, the enzymes involved in pl-CSA synthesis are unclear. Existing researches have confirmed that ChSy-2 has a higher glycosyltransferase activity for CSA than for other CSs and is also highly expressed in placental [17] and cancerous tissues [18]; thus, it is involved in CSA production $[29,30]$. According to the above thesis, we speculated that ChSy-2 may be the key synthase for pl-CSA production. In the present study, the pl-CSA content could be changed through regulating ChSy-2 expression, indicating that ChSy-2 is involved in pl-CSA biosynthesis, which may serve as a potential strategy for therapeutic intervention against glycan dysfunction. However, pl-CSA content could not be wiped out through knockout ChSy-2, suggesting that ChSy-2 was not the unique biosynthetic enzyme. In the process of CSPGs glycosylation, some other enzymes might be involved in pl-CSA biosynthesis, or partly compensated the ChSy-2 function. Meanwhile, this method has disadvantages; for example, the impact of ChSy-2 on tumorigenesis and metastasis prevents the full elucidation of glycan functions. Moreover, in the synthesis or metabolic process of pl-CSA, many enzymes were shown to have synergistic or sequential functions, suggesting that any changes related to any of the enzymes or the core proteins associated with pl-CSA metabolism would result in changes in other enzymes or core protein synthesis, according to the changes in pl-CSA content, to balance or compensate for the effect induced by the pl-CSA alterations. Therefore, the exact mechanism requires further investigation.

Pl-CSA, as a receptor for P. falciparum-infected red blood cells [28], is targeted by the VAR2CSA variant of $P$. falciparum-infected erythrocyte membrane protein 1 with a high affinity and specificity [31]. VAR2CSA, as a transmembrane protein, includes 6 Duffy-binding-like domains (DBLs), in which the minimal binding region is ID1-DBL2Xb with a binding affinity of $21.8 \mathrm{nM}$ [32]. In the present study, a specific binding protein (rVAR2) was also recombined for pl-CSA detection. The signal of rVAR2, labeled with a $6 \times$ His tag, indicating specific binding with pl-CSA, was amplified through a DyLight ${ }^{\circledR}$ 650-labeled anti- $6 \times$ His antibody that showed a high specificity, consistent with previous studies [33]. This may provide an intervention or detection reagents for choriocarcinoma.

$\mathrm{Pl}-\mathrm{CSA}$, also as a pathological polysaccharide, may be an essential component for cell dissociation and invasion, cell-matrix interactions and clustering, as well as angiogenesis and metastasis in tumorigenesis [34]. Over $90 \%$ of tumor tissues, including choriocarcinoma, breast tumor, melanomas, and so on, express pl-CSA on the cell surface and/or in the extracellular matrix (ECM) [14, 35]. The capacities of cancer cells, including proliferation, adhesion, migration and invasion, are tightly associated with ECM, and the assembly and disassembly processes of ECM in cancer cells is required for tumorigenesis and metastasis cycling, suggesting spatiotemporal dynamically expression of specific ECM component of pl-CSA. Our results demonstrate that reduction of pl-CSA content by targeting ChSy-2 inhibited cell proliferation, migration and invasion in vitro, as well as inhibited tumorigenesis and metastasis of choriocarcinoma in vivo, which might be due to the change of the ECM assembly and disassembly processes, also be attributed to abnormal carbohydrate metabolism in tumor cells. Therefore, ChSy-2/pl-CSA system might be promising targets of cancer molecular therapy, but it requires further study.

In conclusion, pl-CSA, as a necessary component for JEG-3 cells, can be efficiently altered by targeting its biosynthetic enzyme ChSy-2. A reduction in pl-CSA content through ChSy-2 knockout inhibited choriocarcinoma tumorigenesis and metastasis through impeding tumor cells capacities of proliferation, migration and invasion. The present results indicated that glycometabolic dysfunction might be a key factor in tumorigenesis and metastasis, and ChSy-2/pl-CSA system might be promising biomarkers for the diagnosis and treatment of choriocarcinoma.

\section{Abbreviations}

ChSy-2: chondroitin sulfate synthase 2; pl-CSA: placental-like chondroitin sulfate A; hCG: human chorionic gonadotropin; ELISAs: enzyme-linked immunosorbent assays; NC: negative control. 


\section{Acknowledgments}

\section{Funding}

This work was supported by grants from the National Key Research and Development Program of China (2018YFC1004103), the Natural Sciences Foundation of China (81830041 and 81771617), the Shenzhen Basic Research Subject Project (JCYJ 20180305164706355, JCYJ20170413165233512, JCYJ 20170817143406822, and JCYJ20180305163428950), the China Postdoctoral Science Foundation (2017M622806), and the Foundation of Hunan Double First-rate Discipline Construction Projects.

\section{Authors' contributions}

J.Z. and Z.C.: conceptualization, investigation, methodology, performance, visualization and writing original draft. J.Z., S.C. and X.F.: conceptualization, writing-reviewing and editing, supervision and project administration. B.W., J.C. and T.X.: preparation of animal cancer models, samples collection, storage, investigation and writingreviewing.

\section{Ethics approval}

All experiments using human cell lines were approved by the Human Ethical Committee of Shenzhen Institutes of Advanced Technology (Shenzhen: SIAT-IRB-180215-H0212). All animal studies were approved by the Animal Ethical and Welfare Committee of Shenzhen Institutes of Advanced Technology (SIAT-IRB-170223-YYS-ZJZA0253-01; Shenzhen, China).

\section{Competing Interests}

The authors have declared that no competing interest exists.

\section{References}

1. Altieri A, Franceschi S, Ferlay J, et al. Epidemiology and aetiology of gestational trophoblastic diseases. Lancet Oncol. 2003; 4: 670-8.

2. Zhang $\mathrm{W}$, Liu $\mathrm{B}, \mathrm{Wu}$ J, et al. Hemoptysis as primary manifestation in three women with choriocarcinoma with pulmonary metastasis: a case series. J Med Case Rep. 2017; 11: 110.

3. Schrader E, Stephens AJ, Shroff $\mathrm{S}$, et al. Widespread choriocarcinoma metastases from de-differentiated gastro-esophageal junction primary adenocarcinoma: A case report with literature review. Gynecol Oncol Rep. 2020; 31: 100513

4. Mangla M, Singla D, Kaur $\mathrm{H}$, et al. Unusual clinical presentations of choriocarcinoma: A systematic review of case reports. Taiwan J Obstet Gynecol. 2017; 56: 1-8.

5. Jiao L, Ghorani E, Sebire NJ, et al. Intraplacental choriocarcinoma: Systematic review and management guidance. Gynecol Oncol. 2016; 141: 624-31.

6. Cheung AN, Zhang HJ, Xue WC, et al. Pathogenesis of choriocarcinoma: clinical, genetic and stem cell perspectives. Future Oncol. 2009; 5: 217-31.

7. Murshed KA, Kanbour A, Akhtar M, et al. Primary mediastinal choriocarcinoma presenting as cutaneous metastasis with resistance to chemotherapy: case report and literature review. J Cutan Pathol. 2020. doi:10.1111/cup.13777; [Online ahead of print].

8. Elzamly S, Torabi A, Padilla O. Testicular Choriocarcinoma Metastasizing to the Small Bowel Causing Intussusception: Case Report. J Gastrointest Cancer. 2019; 50: 1005-8.
9. Wang D, Shu $H$, Zhang $Q$, et al. Brain metastasis of choriocarcinoma presenting as multiple intracranial hematomas: A case report. Medicine (Baltimore). 2018; 97: e12275.

10. Yamanari T, Sawamura M, Lee HJ, et al. Acquired Pulmonary Arteriovenous Fistula within Metastasis from Choriocarcinoma: A Case Report. Case Rep Oncol. 2017; 10: 671-5.

11. Wang JM, Zhao HX, Wang L, et al. The human leukocyte antigen G promotes trophoblast fusion and beta-hCG production through the Erk1/2 pathway in human choriocarcinoma cell lines. Biochem Biophys Res Commun. 2013; 434 : 460-5.

12. Ricciardelli C, Mayne K, Sykes PJ, et al. Elevated stromal chondroitin sulfate glycosaminoglycan predicts progression in early-stage prostate cancer. Clin Cancer Res. 1997; 3: 983-92.

13. Salanti A, Clausen TM, Agerbaek MO, et al. Targeting Human Cancer by a Glycosaminoglycan Binding Malaria Protein. Cancer Cell. 2015; 28: 500-14.

14. Zhang J, Sun B, Zhang K, et al. Screening and surveillance of multiple solid tumours using plasma placental-like chondroitin sulfate A (pl-CSA). Int J Med Sci. 2020; 17: 161-9.

15. Mikami T, Kitagawa H. Biosynthesis and function of chondroitin sulfate. Biochim Biophys Acta. 2013; 1830: 4719-33.

16. Izumikawa $T$, Uyama $T$, Okuura $Y$, et al. Involvement of chondroitin sulfate synthase- 3 (chondroitin synthase-2) in chondroitin polymerization through its interaction with chondroitin synthase-1 or chondroitin-polymerizing factor. Biochem J. 2007; 403: 545-52.

17. Kitagawa H, Izumikawa $\mathrm{T}$, Uyama $\mathrm{T}$, et al. Molecular cloning of a chondroitin polymerizing factor that cooperates with chondroitin synthase for chondroitin polymerization. J Biol Chem. 2003; 278: 23666-71.

18. Kalathas D, Theocharis DA, Bounias D, et al. Chondroitin synthases I, II, III and chondroitin sulfate glucuronyltransferase expression in colorectal cancer. Mol Med Rep. 2011; 4: 363-8.

19. Cao L, Chen $\mathrm{C}$, Zhu H, et al. MMP16 is a marker of poor prognosis in gastric cancer promoting proliferation and invasion. Oncotarget. 2016; 7: 51865-74.

20. Cory G. Scratch-wound assay. Methods Mol Biol. 2011; 769: 25-30.

21. Marshall J. Transwell((R)) invasion assays. Methods Mol Biol. 2011; 769: 97-110.

22. Borowicz S, Van Scoyk M, Avasarala S, et al. The soft agar colony formation assay. J Vis Exp. 2014: e51998.

23. Poussard $\mathrm{A}$, Patterson $\mathrm{M}$, Taylor $\mathrm{K}$, et al. In vivo imaging systems (IVIS) detection of a neuro-invasive encephalitic virus. J Vis Exp. 2012: e4429.

24. Workman P, Aboagye EO, Balkwill F, et al. Guidelines for the welfare and use of animals in cancer research. Br J Cancer. 2010; 102: 1555-77.

25. Nadhan R, Vaman JV, Nirmala C, et al. Insights into dovetailing GTD and Cancers. Crit Rev Oncol Hematol. 2017; 114: 77-90.

26. Zhang B, Cheng G, Zheng M, et al. Targeted delivery of doxorubicin by CSAbinding nanoparticles for choriocarcinoma treatment. Drug Deliv. 2018; 25: 461-71.

27. Ohtsubo K, Marth JD. Glycosylation in cellular mechanisms of health and disease. Cell. 2006; 126: 855-67.

28. Fried M, Duffy PE. Adherence of Plasmodium falciparum to chondroitin sulfate A in the human placenta. Science. 1996; 272: 1502-4.

29. Herman D, Leakey TI, Behrens A, et al. CHST11 gene expression and DNA methylation in breast cancer. Int J Oncol. 2015; 46: 1243-51.

30. Nadanaka S, Kinouchi $\mathrm{H}$, Kitagawa H. Histone deacetylase-mediated regulation of chondroitin 4-O-sulfotransferase-1 (Chst11) gene expression by Wnt/beta-catenin signaling. Biochem Biophys Res Commun. 2016; 480: 234-40

31. Srivastava A, Gangnard S, Round A, et al. Full-length extracellular region of the var2CSA variant of PfEMP1 is required for specific, high-affinity binding to CSA. Proc Natl Acad Sci U S A. 2010; 107: 4884-9.

32. Clausen TM, Christoffersen $S$, Dahlback $M$, et al. Structural and functional insight into how the Plasmodium falciparum VAR2CSA protein mediates binding to chondroitin sulfate A in placental malaria. J Biol Chem. 2012; 287: 23332-45.

33. Sugiura N, Clausen TM, Shioiri T, et al. Molecular dissection of placental malaria protein VAR2CSA interaction with a chemo-enzymatically synthesized chondroitin sulfate library. Glycoconj J. 2016; 33: 985-94.

34. Pinho SS, Reis CA. Glycosylation in cancer: mechanisms and clinical implications. Nat Rev Cancer. 2015; 15: 540-55.

35. Khazamipour N, Al-Nakouzi N, Oo HZ, et al. Oncofetal Chondroitin Sulfate: A Putative Therapeutic Target in Adult and Pediatric Solid Tumors. Cells. 2020; 9(4): 818. 